\title{
Levantamento da freqüência de baixo peso de nascimento em Manaus, 1976
}

\author{
Rodolfo Giugliano $\left({ }^{*}\right)$; José Viana de Souza $\left({ }^{*}\right)$ ); Roger Shrimpton $\left({ }^{* *}\right)$; Loreny Gimenes Giugliano (***)
}

Resumo

Foi levantacio o peso de nascimento de 4.166 crianças nascidas em Manaus, no ano de 1976, em 2 maternidades que atendem a populações de niveis sócio-econômicos diferentes. Na maternidade I (classe pobre), foram levantados 2.552 nascimentos e encontrou-se uma frequiēncia de baixo peso de nascimento $(\leqslant 2,500 \mathrm{~g})$ de $11,7 \%$ enquanto que, na maternidade II (classe média/alta) apurou-se 1.614 nascimentos, com uma freqüência de baixo peso de $8,1 \%$. A diferença foi significativa $(p<0,001)$. Quando analisada a frequência de peso abaixo de $2.000 \mathrm{~g}$. nas duas maternidades, verificou-se que é 4 vezes maior na maternidade $I$ em relação à maternidacle II. São discutidas também comparações desses dados com outros disponiveis na literatura, suas implicaçôes sobre as taxas de mortalidade infantil e o posterior desenvolvimento saudável da criança e as possiveis causas e sugestões para sua melhoria

\section{INTRODUÇÄO}

A OMS (1961) definiu o termo "baixo peso de nascimento" para as crianças nascidas com peso igual ou inferior a $2.500 \mathrm{~g}$, passando-se então a utilizar sua freqüência como um dos indicadores do estado de saúde e nutricionai de uıma comunidade. Esse parâmetro sofre criticas quando consideramos a impossibilidade de utilizá-lo em áreas rurais, porém ele pode ser aplicado em áreas urbanas (Beaton \& Bengoa, 1976) .

O presente levantamento foi motivado pelo fato de que, numa pesquisa anterior que realizamos avaliando o estado nutricional em lactentes de um bairro pobre de Manaus, verificamos que $25 \%$ das crianças examinadas no 1 ? mês de vida tinham peso inferior a $2.500 \mathrm{~g}$ (Shrimpton \& Giugliano, 1977), e também pelo fato de que a mortalidade perinatal concorreu com $37 \%$ da mortalidade infantil em Manaus em 1976 (Giugliano et al., 1978b). A mortalidade perinatal, como sabemos, está diretamente associada com o desenvolvimento intra uterino.

A freqüência de baixo peso de nascimento na Amazônia é desconhecido, pois, num único estudo, sobre peso e estatura de recém-nascidos, feito em Belém (Ferreira, 1961), foram excluídas as crianças nessa faixa de peso. Assim decidimes levantar sua freqüência numa área urbana, a fim de melhorar o nosso conhecimento sobre o estado nutricional do homem na Amazônia.

\section{MATERIAIS E MÉTODOS}

O presente trabalho corresponde à análise 4.166 nascimentos ocorridos em duas maternidades de Manaus, no ano de 1976, coletandose, nos berçários, peso de nascimento, sexo, tipo de parto (normal ou cesariana) e eventuais óbitos.

As duas maternidades escolhidas assim se caracterizam: Maternidade Ana Nery (Maternidade I) - pertence à rede hospitalar do Estacio e atende pacientes de baixo padrão sócio-econômico, com $70 \%$ da amostragem estudada sem direito ao INAMPS (classe pobre). Os dados foram coletados do livro de berçário, anotando-se os nascimentos ocorridos nos meses de janeiro, abril, julho e outubra de 1976 , correspondendo a um total de 2.552 crianças. $\mathrm{O}$ número de atendimentos nessa maternidade é intensivo, com uma média de 637,7 nascimentos/mês. Maternidade Beneficência Portuguesa (Maternidade II) - atende a pacientes particulares e a convênios com INAMPS, comércio, indústria, bancos, etc. (classe média/ alta), e os dados foram coletados dos nascimentos ocorridos nos meses de janeiro a julho

\footnotetext{
(*) - Instituto Nacional de Pesquisas da Amazônia e Instituto de Medicina Tropical de Manaus.

(*) - Acadêmico de Medicina da Fundação Universidade do Amezonas e bolsista do Instituto Nacional de Pesquisas da Amazônia.

(**) - Instituto Nacional de Pesquisas da Amazônia, Manaus.
} 
e outubro de 1976, correspondendo a um total de 1.614 crianças. A média de atendimento nessa Maternidade é de 201,7 nascimentos/ mês.

A idade materna foi constatada em uma subamostra de $5 \%$, ou seja, 265 mães, escoIhidas por sorteio, em ambas maternidades.

Segundo informações da Secretaria de Saúde local, o número de nascidos vivos em Manaus, em 1976, foi de 17.231, crianças e, apesar da precariedade dessas informações, pela ocorrência freqüente de sub-registro de nascimentos, a nossa amostragem correspondeu a cerca de $25 \%$ das crianças nascidas em Manaus nesse ano.

A freqüência de baixo peso de nascimento foi comparada nas duas maternidades, utilizando-se o teste do qui-quadrado (Armintage, 1974).

\section{RESUltados E DISCUSSÃo}

$\mathrm{Na}$ tabela 1 está anotada a distribuição das criariças por faixa de peso em ambas maternidades.

TABELA 1 - Levantamento de peso de nascimento em crianças da população urbana de Manaus, Amazonas, 1976.

\begin{tabular}{|c|c|c|c|c|}
\hline \multirow{2}{*}{$\begin{array}{c}\text { PESO DE } \\
\text { NASCIMENTO } \\
\text { (g) }\end{array}$} & \multicolumn{2}{|c|}{$\begin{array}{l}\text { MATERNIDADE I } \\
\text { (classe pobre) }\end{array}$} & \multicolumn{2}{|c|}{$\begin{array}{l}\text { MATERNIDADE II } \\
\text { (classe média/alta) }\end{array}$} \\
\hline & N. 0 & $\%$ & N. 0 & $\%$ \\
\hline$<2.000$ & 108 & 4,2 & 17 & 1,1 \\
\hline $2.000-2.500$ & 188 & 7,5 & 113 & 7,0 \\
\hline $2.501-3.000$ & 789 & 30,9 & 377 & 23,3 \\
\hline $3.001-3.500$ & 953 & 37,3 & 643 & 39,8 \\
\hline $3.501-4.000$ & 411 & 16,1 & 366 & 22,7 \\
\hline$>4.000$ & 103 & 4,0 & 98 & 6,1 \\
\hline TOTAL & 2.552 & 100,0 & 1.614 & 100,0 \\
\hline
\end{tabular}

Observa-se que na maternidade I (classe pobre), a freqüência de baixo peso de nascicimento $(\leq 2.500 \mathrm{~g})$ atingiu a $11,7 \%$, contra $8,1 \%$ na maternidade II (classe média/alta), sendo a diferença significativa $(p>0,001)$.

Para alguns autores (Beaton \& Bengoa, 1976), a freqüência de peso de nascimento $<2.000 \mathrm{~g}$ é um indicador mais preciso do es- tado de saúde e nutrição de uma comunidade, e nessa faixa de peso a freqüência foi 4 vezes maior na maternidade I do que na maternidade II. Quando comparamos a freqüência do chamado "peso deficiente" (2.500-3.000g) (Puffer \& Serrano, 1975), a diferença é notável, sendo de $30,9 \%$ na maternidade I para $23,3 \%$ na maternidade II. Se, agora, considerarmos a faixa de peso que tem a melhor esperança de vida ao nascer $(3.501-4.000 \mathrm{~g})$ (Puffer \& Serrano, 1975) verificamos que na maternidade I apenas $16,1 \%$ das crianças nasceram nessa faixa, contra $22,7 \%$ na maternidade $\mathrm{II}$.

Esses fatos evidenciam a grande importância do fator sócio-econômico sobre o peso de nascimento e, conseqüentemente, sobre a esperança de vida ao nascer, considerando-se principalmente a agressividade do meio ambiente no qual essas crianças irão viver.

Comparando-se nossos resultados com o Nordeste do Brasil e outras partes do mundo (Tabela 2), verificamos que a freqüência de baixc peso de nascimento da população pobre de Manaus é o dobro dos países desenvolvidos e $2 / 3$ dos valores encontrados no Nordeste. Essa freqüência é comparável com a encontrada em outras áreas subdesenvolvidas como a Nigéria e o Iran, e é ultrapassada por freqüências elevadas nas áreas pobres da India e Indonesia. Se agora compararmos a freqüência de peso de nascimento $<2.000 \mathrm{~g}$ verificamos que o achado em Manaus supera os níveis da população pobre do Iran e se apróxima dos níveis da Índia e Indonésia. Devemos ainda salientar que, em estudo recente que realizamos em um bairro pobre de Manaus (Shrimp. ton \& Giuglianu, 1977), cerca de $40 \%$ das crianças nasceram em casa, não constando portanto de levantamentos em maternidades, e que nessa amostragem $25 \%$ das crianças no 1 o mês tinham peso $\leq 2.500 \mathrm{~g}$.

Quanto às implicações desses fatos, elas já se fazem sentir na própria maternidade. onde constatamos que a mortalidade no berçário atingiu a $17,2 \%$ das crianças com baixo peso na maternidade 1 e $4,3 \%$ na maternidade II (Tabela 3) . Isto evidencia a importância dos cuidados especiais para a sobrevivência dessas crianças. Para as crianças nascidas acima de $2.500 \mathrm{~g}$ não houve diferença na mortalidade

Giugliano et al. 
nas duas maternidades, mostrando que podemos diminuir a mortalidade perinatal melhorando as condiçöes que favoreçam um maior peso de nascimento, mesmo sem a melhoria das condições técnicas de atendimento nas maternidades.

TABELA 2 - Freqüência de baixo peso de nascimento. Comparação dos dados de Manaus com outros levantamentos nacionais e internacionais.

\begin{tabular}{|c|c|c|c|c|}
\hline Lo c a 1 & $\begin{array}{l}\text { População } \\
\text { estudada }\end{array}$ & $\begin{array}{l}<2 \\
\mathbf{K g} \\
\%\end{array}$ & $\begin{array}{l}<2,5 \\
\mathbf{K g} \\
\%\end{array}$ & Referência \\
\hline $\begin{array}{l}\text { India } \\
\text { (Madras) }\end{array}$ & $\mid \begin{array}{l}\text { Próspero } \\
\text { Muito pobre }\end{array}$ & $\begin{array}{l}2 \\
7\end{array}$ & $\begin{array}{l}11 \\
35\end{array}$ & $\begin{array}{l}\text { 3eaton \& } \\
\text { Bengoa, } 1976\end{array}$ \\
\hline Iran & $\begin{array}{l}\text { Próspero } \\
\text { Pobre }\end{array}$ & $\begin{array}{l}0-2 \\
6-7\end{array}$ & $\begin{array}{c}8 \\
21-26\end{array}$ & $n$ \\
\hline Indonésia & $\begin{array}{l}\text { Próspero } \\
\text { Pobre }\end{array}$ & $\begin{array}{l}1,9 \\
3,9\end{array}$ & $\begin{array}{l}10,7 \\
14,6\end{array}$ & $n$ \\
\hline Grä-Bretanha & $\begin{array}{l}\text { População } \\
\text { Geral }\end{array}$ & - & 5.8 & $n$ \\
\hline $\begin{array}{l}\text { Rep. Democr. } \\
\text { da Alemanha }\end{array}$ & $\begin{array}{l}\text { População } \\
\text { Geral }\end{array}$ & 2,3 & 6,0 & n \\
\hline Nigéria & $\begin{array}{l}\text { Urbana } \\
\text { Rural }\end{array}$ & $\begin{array}{l}0,9 \\
1,6\end{array}$ & $\begin{array}{l}9,4 \\
9,8\end{array}$ & $\begin{array}{l}\text { Oduntan } \\
\text { et al., } 1977\end{array}$ \\
\hline Manaus & $\begin{array}{l}\text { Próspero } \\
\text { Pobre }\end{array}$ & $\begin{array}{l}1,1 \\
4,2\end{array}$ & $\begin{array}{r}8,1 \\
11.7 \\
\end{array}$ & $\left({ }^{\circ}\right)$ \\
\hline Recife & $?$ & $?$ & 15,0 & $\begin{array}{l}\text { Puffer \& } \\
\text { Serrano, } \\
1975\end{array}$ \\
\hline
\end{tabular}

(*) - Presente levontamento.

TABELA 3 - Mortalidade no berçário relacionada ao peso de nascimento em 2 maternidades de Manaus, Amazonas, 1976

\begin{tabular}{|c|c|c|c|c|c|c|}
\hline \multirow{2}{*}{ Peso $(\mathrm{g})$} & \multicolumn{3}{|c|}{ Maternidade 1} & \multicolumn{3}{|c|}{ Maternidade II } \\
\hline & N. & óbitos & $\%$ & N. & óbitos & $\%$ \\
\hline$\leqslant 2.500$ & 314 & 54 & 17,2 & 139 & 6 & 4,3 \\
\hline $2.501-3.000$ & 776 & 4 & 0,5 & 369 & 1 & 0.3 \\
\hline$>3.000$ & 1.462 & 5 & 0,3 & 1.106 & 2 & 0,2 \\
\hline
\end{tabular}

As influências negativas do baixo peso de nascimento sobre o futuro do indivíduo têm sido constatadas em vários trabalhos. Em recente estudo da O. P. S. (Puffer \& Serrano, 1975), verificou-se que o peso de nascimento, a idade materna e a ordem de nascimento são 3 importantes fatores determinantes da mortalidade infantil nas Américas. No mesmo trabalhe concluiu-se que a mortalidade infantil é menor nas crianças com peso de nascimento entre $3.501-4.000 \mathrm{~g}$, filhos de mães com idade entre 25-29 ancs. Quanto à ordem de nascimento, os menores índices de mortalidade foram para o $1^{\circ}$ filho, aumentando sensivelmente a partir do $4 \%$ filho.

Outro aspecto desfavorável do baixo peso de nascimento, uma forma de desnutrição precose, são suas implicações sobre o desenvolvimento neurológico da criança. Esse assunto tem sido muito estudado atualmente e Winick, citado por Cheek et al. (1972), concluiu, através de estudos em animais e humanos, que, no período crítico de crescimento cerebral, a malnutrição, mesmo nas formas médias e por curto período, pode produzir danos irreversíveis. Como sabemos, o período crítico de crescimento cerebral ocorre antes do nascimento e nos primeiros meses de vida pós-natal. Dobbing (1974) refere, porém, que ainda são pouco conhecidas as bases físicas da atividade mental e que parece que se correlacionam mais com o sistema de ramos dendríticos e conecções sinápticas do que com o número de células. Nobrega (1974) cita o encontro de de deficiências consideráveis do quociente intelectual (QI), em estudos longitudinais de crianças com peso de nascimento $\leqslant 2.500 \mathrm{~g}$.

A influência do baixo peso de nascimento sobre o setor imunológico de criança recebeu destaque, ultimamente, quando estudos mostraram que elas apresentam maior susceptibilidade às infecções e septicemias por bacterias Gram - que os níveis de IgG recebidos da mãe são menores do que em crianças de peso normal (Faulk et al., 1975) e também por uma imunidade celular que pode permanecer deficiente por vários meses após o nascimento (Effect of Fetal Growth..., 1977).

A anemia é um problema seríssimo em nossa região (Giugliano et al., 1978a), e são conhecidas as baixas reservas de ferro dessas crianças (Bowering \& Sanches, 1976), predispondo-as a uma anemia precoce. O problema se agrava quando sabemos que os niveis de $\mathrm{Fe}$ sérico, $\mathrm{Hb}$ e $\mathrm{Htc}$ fetais são menores em fi- 
Ihos de gestantes anêmicas, mesmo sem considerar o peso de nascimento (Szarfac, 1975).

Perante todos esses fatos, é notória a precariedade com que essas crianças nascem, considerando-se principalmente o meio agressivo onde vão se desenvolver.

Quanto aos fatores que influenciam o peso de nascimento, Drillien (1975) e Wehmer \& Hafez (1975) referem a altura e idade maternas, ganho médio de peso na gravidez, estado nutricional materno antes e durante a gravidez. fatores sócio-econômicos, estado marital da mãe, idade gestacional, multiparidade, gravidez patológica, tabagismo, grupo étnico e fatores genéticos. Salientaremos desta lista as infecções maternas, que, de maneira relevante, influenciam o peso de nascimento (Mata, 1975; Beisel, 1975), especialmente em áreas subdesenvolvidas como a Amazônia.

No que se refere aos fatores étnico-genéticos, Leitch (1976) cita uma tendência dos filhos de ameríndios e esquimós a terem um peso maior de nascimento que os europeus, apesar das mães não serem maiores do que as mulheres européias. Malcolm (1973), em publicação referente ao fatores ecológicos relacionados ao crescimento e estado nutricional. conclui que :

...० peso de nascimento é determinado $\mathrm{cm}$ larga extensão pela qualidade do meio ambiente total antes do nascimento mais que o genótipo de ambos, pais e feto; e que essa experiência biológica precoce pode permanecer influenciando os padrôes de crescimento subseqüente da criança, levando a impressão de um ultimato sobre as características do adulto.

Assim, parece que as tendêricias atuais consideram que o fator ambiental atua de maneira mais importante sobre o peso de nascimento do que o étnico-genético nas áreas subdesenvolvidas.

Nessas áreas, as causas implicadas com o baixo peso de nascimento confundem-se com as c:ausas gerais da desnutrição, ressaltandose a baixa renda, más condiçōes higiênico-sanitárias, deficiências educacionais e alguns problemas específicos, como a maternidade precoce e a falta de assistência pré-natal. $\mathrm{Na}$ região amazônica, a maternidade precoce é um fato muito freçüente, sendo comum a gravidez antes dos 20 anos e as conhecidas "mäes solteiras". Em nosso estudo, quando comparamos a média de idade materna nas duas maternidades, verificamos ser ela bem inferior na maternidade $\mathrm{I}$, onde atinge a $22,4 \pm 4,8$ anos, contra $26,8 \pm 5,2$ anos na maternidade II. A freqüência de mães com idade $₹ 20$ anos foi de $54,2 \%$ e $7,8 \%$, respectivamente. A importância da idade materna na freqüência de baixo peso de nascimento pode ser verificado na tabela 4. onde a porcentagem de recém-nascidos de baixo peso nas mães com idade igual ou inferior a 20 anos foi $\alpha$ dobro de todas as outras idades em conjunto.

TABELA 4 - Freqüência de baixo peso de nascimento de 31 crianças escolhidas ao acaso relacio. nada a idade materna. Manaus, Amazonas, 1976.

\begin{tabular}{c|c}
\hline Idade materna & $\begin{array}{c}\text { Frẹüência de baixo } \\
\text { peso }\end{array}$ \\
$\leqslant 20$ & $17(54,8 \%)$ \\
$21-25$ & $6(19,4 \%)$ \\
$26-30$ & $5(16,1 \%)$ \\
$>30$ & $3(9,7 \%)$ \\
\hline TOTAL & $31 \quad 100,0 \%$ \\
\hline
\end{tabular}

A influência do sexo sobre a distribuição de crianças na faixa de baixo peso de nascimento não foi significativa, como podemos verificar na tabela 5 .

TABELA 5 - Distribuição por sexo de 427 crianças nascidas com baixo peso em 2 maternidades de Manaus, Amazonas, 1976.

\begin{tabular}{|c|c|c|c|c|}
\hline \multirow{2}{*}{ Sexo } & \multicolumn{2}{|c|}{ Maternidade I } & \multicolumn{2}{|c|}{ Materniciade II } \\
\hline & N. & $\%$ & N. & $\%$ \\
\hline Masculino & 152 & $51,2 \%$ & 62 & $47,7 \%$ \\
\hline Feminino & 145 & $48,8 \%$ & 68 & $52,3 \%$ \\
\hline TOTAL & 297 & $100,0^{\%} \%$ & 130 & $100,0 \%$ \\
\hline
\end{tabular}


As infecções maternas também desempenham um papel importante sobre a freqüência de baixo peso de nascimento, sendo conhecidos os efeitos negativos de viroses (rubéola, citomegalovírus, hepatite), sifilis, tuberculose, malária e toxoplasmose (Mata, 1975; Beisel, 1975). Algumas dessas doenças têm comprovadamente prevalências elevadas na região, como hepatite, sífilis, tuberculose, malária e toxoplasmose, conforme observaçỏes pessoais e levantamentos estatísticos e sorológicos recentes (Giugliano et al., 1978 b; Ferraroni, 1977 e Ferraroni \& Marzochi, 1978).

O estado nutricional materno exerce influênccia marcante sobre o peso de nascimento. Não dispomos de levantamentos sobre o estado nutricional e ganho médio de peso de gestantes nesta regiāo, porém, em trabalho recente sobre o consumo de alimentos e nutrientes em Manaus (Shrimpton \& Giugliano, 1979), foi constatado que o consumo proteico excede em muito as necessidades per capita/dia, devido ao elevado consumo de peixe na região. As deficiências marcantes indicadas na dieta local são de vitamina $A$, riboflavina e, provavelmente, tiamina, associada ao baixo consumo de frutas, verduras e legumes. A mesma dieta mostrou-se também deficiente em Zinco (Shrimpton, comunicações pessoal), elemento traço muito estudado atualmente. O consumo energético mostrou-se próximo da normalidade, porém, considerando-se as dificuldades na sua avaliação, poderão ocorrer deficiências na distribuição intrafamiliar. O consumo de ferro atingiu as necessidades per capita/dia recomendadas, porém a anemia é um problema extremamente sério na região, devido à grande incidência local de verminose, principalmente a ancilostomose (Giugliano et al., 1978b) .

Os efeitos do estado nutricional materno sobre o crescimento fetal parecem ser maiores na presença de desnutrição crônica materna do que na aguda, e em nossa região prevalece a desnutrição crônica (Shrimpton \& Giugliano, 1977; Giugliano \& Shrimpton, 1977 e Giugliano et al., 1978a). Estudos com suplementaçăo calórica em gestantes de áreas onde prevalece a desnutriçăo crônica mostram bons resultados (Frisancho et al., 1977). A suplementação com niveis superiores a 20.000 calorias no período gestacional levou a considerável redução na freqüência de baixo peso de nascimento (Klein et al., 1976). Na Guatemala, verificou-se uma correlação da melhora no peso de nascimento com o valor calórico total da dieta, mas não com o conteúdo de proteínas e carboidratos (The influence of maternal.... 1976). Foi notado também que a suplementação calórica deve ser mais intensiva no último trimestre da gravidez, e os resultados são melhores quando à mesma se associa o combate à anemia e ao enteroparasitismo (Rosa \& Turshen, 1970). É fundamental, no entanto, para a implantação de um programa de suplementação de gestantes, o conhecimento prévio das deficiências e hábitos dietéticos locais (Lechtig, 1975).

São poucos ainda os dados sobre deficiências específicas influenciando o peso de nascimento, porém sabe-se que a freqüência de baixo peso de nascimento é maior em mães anêmicas (WHO, 1975), e que em áreas carentes em zinco é maior a natimortalidade (Sever. 1975), faltando ainda estudos sobre suas correlações com o peso de nascimento. Em regiões com dieta deficiente em Vitamina A têm sido referido níveis baixos de retinol no sangue de cordão, menores do que os níveis maternos, sendo ainda desconhecidas as implicações desse fato (Arroyave et al., 1975). A tendência, na atualidade, é afirmar que o peso de nascimento se correlaciona mais com a qualidade total da dieta do que com deficiências específicas (Philipps \& Johnson, 1977).

TABELA 6 - Distribuição por tipo de parto em 4.166 nascimentos ocorridos em 2 maternidades de Manaus, Amazonas, 1976.

\begin{tabular}{|c|c|c|c|c|c|}
\hline \multirow{2}{*}{ Tipo de parto } & \multicolumn{2}{|c|}{ Normal } & \multicolumn{2}{|c|}{ Cesariana } & \multirow[b]{2}{*}{ Tot a 1} \\
\hline & N. & $\%$ & N. & $\%$ & \\
\hline Maternidade I & 2.237 & 87,7 & 315 & 12,3 & 2.552 \\
\hline Maternidade II & 1.126 & 69,8 & 488 & 30,2 & 1.614 \\
\hline
\end{tabular}

A dieta em Manaus parece satisfatória do ponto de vista quantitativo, porém, em termos qualitativo, é possivelmente uma das piores do país (Shrimpton \& Giugliano, s.d.). 
Por último, é importante observarmos a frequiência de parto cesária que atingiu a $30,2 \%$ na maternidade II, contra $12,3 \%$ da maternidade I (Tabela 6), cuja diferença atribuímos à condenavel cesária com "data marcada" ou com finalidades de "laqueadura de cordão", as quais, evidentemente, não são indicações médicas para uma cesariana. Os perigos de "fazermos nascer" crianças com baixo peso de nascimento não são desprezíveis.

\section{CONCLUSŌES E RECOMENDAÇÕES}

A freqüência de baixo peso de nascimento na população pobre de Manaus, atendida em maternidade, atingiu a $11,7 \%$, valor já considerável se compararmos com dados do Nordeste e de outras regiões subdesenvolvidas do mundo. Essa freqüência pode ser bem superior se considerarmos que cerca de $40 \%$ das mães pobres da cidade têm filhos em casa e, portanto, não estão incluídas nessa estatística. As principais causas locais de baixo peso de nascimento são a maternidade precoce, alta freqüência de infecções, anemia materna e uma dieta qualitativamente deficiente.

As implicações desse fato já se fazem sentir de imediato na elevada mortalidade no berçário e na mortalidade perinatal em Manaus, que atinge cerca de $37 \%$ da mortalidade infantil. A médio e longo prazo, são conhecidas as deficiências dessas crianças na capacidade intelectual, imunitária e uma predisposição à anemia precoce, levando-se em conta, principalmente, $\alpha$ meio agressivo onde elas irão se desenvolver.

Frente a esse problema, faz-se necessário um estudo do estado nutricional, padrões alimentares e ganho médio de peso das gestantes pobres de Manaus, que não foi realizado até o momento.

Abaixo colocamos algumas sugestões para melhoria a curto e médio prazos do problema : 1) Combate à maternidade precoce - campanhas educativas da população em geral e principalmente nas escolas, sobre os perigos da maternidade precoce para mãe e para o feto; 2) Intensificação da assistência pré-natal em recente levantamento que fizemos em um bairro pobre da cidade, cerca de $40 \%$ das mães não freqüentaram serviço de pré-natal. Lembrando a saturação e alto custo dos serviços de pré-natal em maternidades e postos de saúde, acreditamos que a educação de pessoal para-médico (curiosas, líderes de comunidades, etc.) seria a única maneira de atuação em larga escala. O acompanhamento visaria principalmente $\alpha$ combate a anemia, enteroparasitoses e infecções maternas, associado à suplementação alimentar da gestante pobre. Os casos fora da rotina seriam encaminhados a postos de saúde ou maternidades;

3) Suplementação alimentar da gestante pobre - a distribuição de alimentos para gestantes pobres em Manaus é ainda precária. No mesmo levantamento citado acima verificamos que somente $10 \%$ das mães que freqüentaram prénatal receberam alimentos. Os alimentos comumente distribuídos são feijão, arroz (2 $\mathrm{kg} / \mathrm{mês}$ ), farinha seca e açúcar (1 $\mathrm{kg} / \mathrm{mês}$ ).

Considerando-se o recebimento regular desses alimentos nos 9 meses de gestação, e a distribuição intrafamiliar do mesmo que fatalmente ocorre ("), teriamos, mesmo assim, cerca de 30.000 cal a mais para a gestante, o que supera os níveis recomendados por alguns autores (Klein et al., 1976). Em termos calóricos, portanto, o que deve ocorrer é a expansão do programa para atingir um maior número de gestantes. Qualitativamente, recomendaríamos a substituição da farinha seca pela farinha d'água amarela, melhor aceita na região e que contém vitamina A (Maravalhas, 1964). Achamos também que essa suplementação não fornece $\sigma$ que realmente a populaçåo local é carente em consumo, como verduras, frutas e legumes. A suplementação com esses aìimentos deve ser estudada.

\section{AgRADECIMENTOS}

Ao Comendador José Cruz, diretor da Beneficente Portuguesa e ao Dr. Oswaldo Leal Gesta, diretor da Maternidade Ana Nery, pela permissão da realização dos levantamentơs.

\section{SUMMARY}

The Amazon Basin has bern called a zons of endemic hunger, however iow representative scientific studies of human nutritional status have 
been carried out. Birth weight is influenced amongts other things by nutritional status. As data on ths frequency of low birth weights in the Amazon was not available, 4168 birth records for the year 1970 were studied in two maternity ciinics of Manaus. One clinic with 2552 births, attended principally the poor of the city, whilst the second attended 1614 births of a higher socio-economic level. The study represent some $25 \%$ of the estimated total number of births in the city for 1976. The frequency of low birth weight in the poor maternity clinic $(11.7 \%)$ was higher than in the richer one $(8.1 \%)(P<0,001)$ Attention is brought to the fact that most probabiv half of the poorest people in the city had their children at home. In comparison with other parts of the world these frequencies are intermediate between the north east of Brazil and developed countries, being only half of the frequencies found in Asia. The possible local factors responsible for these results are discussend and recommendations made that could improve the situation.

\section{BIBLIOGRAFIA}

ARMintAGE, P.

1974 - Statiscal methods in medical research. London, Blackwell, 504 p.

Arroyave, G.; Moscoso, Y.M. \& Lechtig, A. 1975 - Vitamina A no sangue de embarazadas y sus recien-nascidos de dos grupos socio-economicos. Arch, Latinoam. Nutr., 25(3) : 283-290.

Benton, G.H. \& Bengon, J.M.

1976 - In: Beaton, G.H. \& Bengoa, J.M. Ed. Nutrition in preventive medicine. World Health Organization Monograph Series, $62: 500-519$

BEISEL, W.R.

1975 - Synergistic efeects of maternal malnutrition and infection on the infant. Am. J. Dis. Child, $129: 571-577$.

BOWERING, J. \& SANCHEZ, A.M.

1976 - A conspectus of research on iron requirements of man. The journal of Nutrition, 106(7): 9851074.

Chfek, D.B.; Holt, A.B. \& Mellits, E.D.

1972 - In: Nutrition, the nervous system, and behavior. Pan American Health Organization. Scientific Publication, 251:3-14.

CODEAMA

1976 - Anuário Estatístico 1975. Manaus, Go. = verno do Estado do Amazonas. 298 p.

DoBBing, J.

1974 - In: Almqvist \& Wiksell Ed. - Early malnutrition and mental development. Stockholm, Sweden. p. 96-110.
DRILLIEN, C.M.

1975 - In: Mitchell, R.G. Ed. - Crescimiento y desarrollo del niño. Barcelona, p. 115-130.

EFFECT OF FETAL GROWTH RETARDATION ON IMMUNOLOGIC SATUS

1977 - Nutrition Reviews, 35(2): 41-42.

- Faulk, P.W.; Mata. L.J. \& Edsall, G.

1975 - Effects of malnutrition on the immune response in humans: A Review. Tropical Diseases Bulletin, 72(2): 89-103.

FERraroni, J.J,

1977 - A possivel incidência da sifilis em gestantes da cidade de Manaus. Acta Amazonica, $7(2): 255-262$.

FERRARONI, J.J. \& MARZOCHI, M.C.A.

1978 - Toxoplasmose em animais domésticos e silvestres de Manaus-Amazonas. Acta Amazonica, 8(1) : 83-89.

FERrEIRA, M.R.

1961 - Peso e estatura de recém-nascidos em Belém. Tese de livre Docência, Universidade do Pará. 60 p.

Frisancho, A.R.; Klayman, J.E. \& Matos, J.

1977 - Influence of maternal nutritional status on pré-natal growth in a peruvian urban population. Am. J. Phys. Anthrop., $64(2): 265-274$

Giuglino, R.; Albuquerque, H.C.R. \& Shrimpton, R. 1978a - Estudo antropométrico, clinico e de padrōes alimentares em um grupo de escolares de Manaus. Acta Amazonica, $8(1): 75-82$

GiUgliano, R. \& Shrimpton, R.

1977 - Estudo antropométrico e clínico do estado nutricional em um grupo de crianças pré-escolares de Manaus. Acta Ama. zonica, $7(2): 389-394$.

Giugliano, R.; Shrimpton, R.; ARCKoll, D.B.; Giugliano, L.G. \& Petrere Junior, M.

1978b - Diagnóstico da realidade alimentar $e$ nutricional do estado do Amazonas. Acta Amazonica, 8(2) : Suplem, 2.

Klein, R.E.; Arenales, P.; Delgado, H.; Eugle, P.L.; GuzMAN, G.; Irwin, M.; LASKY, R.; Lechtig, A.; Martorell, R.; pivaral, V.M.; Russel, P. \& YARBROUGH, C.

1976 - Effects of maternal nutrition on fetal growth and infant development. Bull. Pan. Am. Health. Organ, 10(4) : 301-316.

lechtig, A.; Yarbrough, C.; Delgado, H.; Habicht, J.P.; MARTORELl, R. \& Klein, R.E.

1975 - Influence of maternal nutrition on birth weight. The American Journal of Clinical Nutrition, $28:$ 1223-1233.

LEITCH, I

1976 - Change in shape of the human body. Progr. Ed. Nutr. Sci. 2 : 99-141. 
MaLCOM, L.A.

1973 - In: Roche, A.F. and Falkner, F. Ed. Advances in Experimental Medicine and Biology, New York and London, 49: 329-354.

Maravalhas, N.

1964 - Cinco estudos sobre a farinha de mandioca. Manaus. Publicação do INPA. Ser. Químico, $4:$ 1-41.

Mata, L.J.

1975 - Malnutrition - Infection interactions in the tropics. Am. J. Trop. Med. \& Hig., 24(4) : 564-574.

NoBrega, F.J.

1974 - Desnutrição e deficiência mental. Jornal de Pediatria, 39(5-6) : 129-135.

Oduntan, S.O.; Odunlami, V.B. \& Ayeni, O.

1977 - The birth weights of nigerian babies.

The Journal of Tropical Pediatrics and

Environmental Child Health, 23(3): 141-144.

Organizacion Mundial de la Salud.

1961 - La insuficiência ponderal del recien nascido desde el punto de vista sanitário - Ser. Inf. Tecn, 217, 19 p.

PhILIPPS, C. \& Johnson, N.E.

1977 - The impact of quality of diet and other factors on birth weight of infants. The American Journal of Clinical Nutrition, $30: 215-225$.

PUffer, R.R. \& SERrano, C.V.

1975 - El peso al nascer, la idade materna y el ordem de nascimiento: Tres importantes determinantes de la mortalidad infantil.
Organizacion Panamericana de la Salud, Publ. Cient. 294, 47 p.

Rosa, F.W. \& TURSHEN, M.

$1970-$ Fetal nutrition. Bull. Wld. Hlth. Org. $43: 785-795$.

SEVER, L.E.

1975 - Zinc and human development: A review. Human Ecology, 3 : 43-57.

SHrimpton, R. \& GiuglinNo, R.

1979 - Consumo de alimentos e alguns nutrientes em Manaus, Amazonas, 1973-4. Acta Amazonica 9(1): 117-142.

Shrimpton, R, \& Giugliano, R.

1977 - Nutriçăo em lactentes de um bairro de Manaus, Amazonas. Acta Amazonica, $7(2): 247-253$.

SZARFAC, S.C.

1975 - Comparação entre valores hematológ1cos (hemoglobina, hematócrito e ferro sérico) da parturiente e do rəcém-nascido. Rev. Saúde Publ., SP 9: 43-47.

THE INFLUENCE OF MATERNAL FOOD SUPLEMENTS ON BIRTH WEIGHT IN GUATEMALA. 1976 - Nutrition Revicws, 34(6) : 169-172.

WEHMER, F. \& HAFEZ, E.S.E.

1975 - In: Hafez, E.S.E. Ed. The Mammalian Fetus, Illinois, p. 154-185.

World Health Organization

1975 - Control of nutritional anaemia with special reference to iron deficiency. Tech. Rep. Ser., 580, $71 \mathrm{p}$.

(Aceito para publicação em 12/02/79) 